\title{
Ethosuximide modifies network excitability in the rat entorhinal cortex via an increase in GABA release
}

\author{
Stuart D. Greenhill ${ }^{\mathrm{a}, \mathrm{c}}$, Nicola H. Morgan ${ }^{\mathrm{b}}$, Peter V. Massey ${ }^{\mathrm{a}}$, Gavin L. Woodhall ${ }^{\mathrm{b}}$ \\ and Roland S.G. Jones ${ }^{a, d}$
}
${ }^{a}$ Department of Pharmacy and Pharmacology, University of Bath, Claverton Down, Bath BA2 7AY, UK

${ }^{b}$ School of Life and Health Sciences, Aston University, Birmingham B4 7ET, UK

\author{
'Present Address: School of Biosciences, Cardiff University, Museum Avenue, \\ Cardiff CF10 3AX, UK. \\ ${ }^{d}$ Corresponding Author: Roland S.G. Jones, Department of Pharmacy and \\ Pharmacology, University of Bath, Claverton Down, Bath, BA2 7AY, UK. \\ Tel: +44-1225-383935 \\ Fax: +44-1225-386114 \\ E-mail: r.s.g.jones@bath.ac.uk
}

Abbreviations: aCSF, artificial cerebrospinal fluid; $E C$, entorhinal cortex; $E_{\mathrm{Bg}}$, global background synaptic excitation; GABA $A_{A} r, G A B A_{A}$-receptor; GABA ${ }_{B} r$, GABA $A_{B}$-receptor; GAERS, Genetic Absence Epilepsy Rat from Strasbourg; GIRK, G-protein couple inwardly rectifying K-channel; I $_{\mathrm{Bg}}$, global background synaptic inhibition; I:E, inhibition:excitation ratio; IEI, inter-event interval; KS, KolmogorovSmirnov test; mIPSC, miniature inhibitory postsynaptic current; sEPSC, spontaneous excitatory postsynaptic current; sIPSC, spontaneous inhibitory postsynaptic current; T-type, low threshold Cachannel; VmD, membrane potential distribution method. 


\begin{abstract}
Ethosuximide is the drug of choice for treating generalised absence seizures, but its mechanism of action is still a matter of debate. It has long been thought to act by disrupting a thalamic focus via blockade of T-type channels and thus generation of spike wave activity in thalamo-cortical pathways. However, there is now good evidence that generalised absence seizures may be initiated at a cortical focus and that ethosuximide may target this focus. In the present study we have looked at the effect ethosuximide on glutamate and GABA release at synapses in the rat entorhinal cortex in vitro, using two experimental approaches. Whole-cell patch clamp-studies revealed an increase in spontaneous GABA release by ethosuximide concurrent with no change in glutamate release. This was reflected in studies that estimated global background inhibition and excitation from intracellularly recorded membrane potential fluctuations, where there was a substantial rise in the ratio of network inhibition to excitation, and a concurrent decrease in excitability of neurones embedded in this network. These studies suggest that, in addition to wellcharacterised effects on ion channels, ethosuximide may directly elevate synaptic inhibition in the cortex and that this could contribute to its anti-absence effects.
\end{abstract}

Key words: entorhinal cortex; ethosuximide; glutamate release, GABA release, excitability 


\section{Introduction}

Generalised, non-convulsive absence seizures are characterised by brief episodes of unconsciousness accompanied by synchronised bilateral spike and wave discharges (SWD). Ethosuximide is the drug of choice in the treatment of absence epilepsy (Posner et al., 2004; Glauser et al., 2010). It has a number of potential molecular targets, but its precise mechanism of action has not been elucidated.

One view of the basis of absence epilepsy is that it involves an interaction between a hyperexcitable cortex and a rhythm generator operative via a thalamocortical loop, where rhythmical bursts of activity in the GABAergic neurones of the thalamic reticular nucleus play a central role (see Crunelli and Leresche, 2002a; Manning et al., 2003; Meeren et al., 2005). These bursts of activity are driven by low-threshold (T-type) Ca-currents, and it has been postulated that block of T-type Ca-channels in thalamocortical reticular nucleus neurones may be the basis of the anti-absence effects of ethosuximide (Coulter et al., 1989a,b; Kostyuk et al., 1992; Huguenard, 1999; Gomora et al., 2001). However, there is still controversy over whether this blockade is the whole story (see Crunelli and Leresche, 2002b; Manning et al., 2003). Certainly, ethosuximide is promiscuous in its effects and has been shown to block other ion channels (Leresche et al., 1998; Kobayashi et al., 2009).

Early studies implicated the thalamus as a primary site of epileptogenesis, but increasing attention has centred on a cortical focus in absence seizures (Siedenbecher et al., 1998; Meeren et al, 2002; 2005; Holmes et al., 2004; Sadlier et al., 2006; Polack et al., 2007). It is possible that ethosuximide acts partially, or exclusively, at this level. A weak and delayed reduction of the spike-wave discharges in genetically epileptic rats followed infusion of ethosuximide directly into the thalamus, but systemic injection resulted in immediate cessation of discharges (Richards et al., 2003). Likewise, direct application into somatosensory cortex caused immediate cessation of discharges (Manning et al., 2004; Gülhan-Aker et al., 2010). Polack et al. (2007) suggested that spike-wave discharges were initiated by pyramidal neurones layer $\mathrm{V}$, and found that hyperexcitability in this region was normalised by systemic ethosuximide (Polack and Charpier, 2009). The molecular mechanism of this action of ethosuximide could involve actions at one or more of the 
ion channel targets noted above but it is also feasible that alterations of inhibitory or excitatory synaptic transmission could be involved. For example, Luhman et al. (1994) showed an impairment of GABA inhibition in the cortex of WAG/Rij rats, a genetic model of absence epilepsy. Also, A number of mutations in $\mathrm{GABA}_{A}$-receptors $\left(G_{B B A} r\right.$ ) have been linked to childhood absences (see Galanopoulou, 2010; McDonald et al., 2010). One mutation, in the $\gamma 2$ subunit, has been shown to elicit ethosuximide-sensitive absence seizures when expressed in mice, associated with a reduced expression of the subunit and a decrease in $\mathrm{GABA}_{A} r$ mediated events in somatosensory cortex (Tan et al., 2007). Thus, cortical hyperexcitability could be associated with the loss of GABA inhibition and ethosuximide could target cortical GABA transmission in absence epilepsy.

We have recently studied the effects of anticonvulsant drugs on excitatory and inhibitory transmission in the entorhinal cortex (EC). Using patch clamp recordings of spontaneous postsynaptic currents, we have studied the effects of the drugs on GABA and glutamate release (Cunningham et al., 2000; 2003; 2004; Cunningham and Jones, 2000; Yang et al., 2007). We have complemented these studies using a novel approach (Greenhill and Jones, 2007; 2010) to determine global, network driven, background excitation and inhibition, estimated from the distribution of resting membrane potential fluctuations $(\mathrm{VmD})$ in individual neurones (see Rudolph et al., $2004 ; 2007)$. We have studied a number of drugs used to treat tonic-clonic convulsive seizures, some of which are also used in absence epilepsies. Patch clamp studies revealed diverse effects on spontaneous of glutamate and GABA release, and these were reflected by global changes in excitation and inhibition determined by VmD studies. However, the drugs had the common effect of elevating the ratio between inhibition and excitation in favour of the former (Greenhill and Jones, 2010). Concurrently, they caused a decline in intrinsic neuronal excitability.

We have now determined the effect of specific anti-absence drug, ethosuximide, on synaptic transmission and excitability in the rat EC. Whilst evidence suggests that the frontal and somatosensory cortex are likely foci in absence seizures (Meeren et al., 2002; Polack et al., 2007), EEG studies indicate that some absence and related seizures could originate at temporal sites (e.g. Lambroso, 1997; Holmes et al., 2010; 
Caraballo et al, 2008; Tucker et al., 2007). In addition, studies in EC-hippocampal slices have suggested that there is altered excitability and susceptibility to epileptogenesis in GAERS (Genetic Absence Epilepsy Rat from Strasbourg; Armand et al, 1998; 1999), and it is also the case that local cerebral glucose utilisation is increased in limbic regions (including EC) as well frontal and somatosensory areas (Nehlig et al., 1998; Carcak et al., 2009) in GAERS. For these reasons, and because our investigations of other anticonvulsants have focussed on this area we decided to continue with it in the present study, although we do not suggest that the EC may be a primary focus in absence seizures

\section{Materials and Methods}

\subsection{Slice preparation}

All experiments were performed in accordance with the U.K. Animals (Scientific Procedures) Act 1986, European Communities Council Directive 1986 (86/609/EEC) and the University of Bath ethical review document. The number of animals used was kept to a minimum and every precaution was taken to minimize any suffering and stress inflicted. EC slices were prepared from male Wistar rats $(60-100 \mathrm{~g})$ male Wistar rats (P28-40) anaesthetised with ketamine $(120 \mathrm{mg} / \mathrm{kg})$ plus xylazine $(8$ $\mathrm{mg} / \mathrm{kg}$ ). Rats were decapitated and the brain removed and immersed in artificial cerebrospinal fluid (aCSF; see below for composition) at $4^{\circ} \mathrm{C}$. Slices ( $\left.400 \mu \mathrm{M}\right)$ were cut using a Campden Vibroslice and stored in aCSF bubbled with carbogen (95\% $\mathrm{O}_{2} / 5 \% \mathrm{CO}_{2}$ ) at room temperature. To increase neuronal survival and viability, ketamine $(4 \mu \mathrm{M})$ was included in the cutting solution, and the antioxidants n-acetyl-Icysteine $(6 \mu \mathrm{M})$ and uric acid $(100 \mu \mathrm{M})$, added to both cutting and storage solutions. For patch clamp recordings slices were transferred to a recording chamber perfused $(2 \mathrm{ml} / \mathrm{min})$ with oxygenated aCSF at $31-32^{\circ} \mathrm{C}$ on an Olympus BX50WI microscope. Neurones were visualized using DIC optics and an infrared video camera. In VmD experiments slices were transferred to a recording chamber where they were held at the interface between a continuous perfusion of oxygenated aCSF $(1.5 \mathrm{ml} / \mathrm{min})$ maintained at $32 \pm 0.5^{\circ} \mathrm{C}$ and warm moist carbogen gas. Intracellular recordings were made "blind" from slices visualised with a binocular microscope (Wild M8). We have found (Woodhall, G.L. and Jones, R.S.G., unpublished observations) that the 
use of antioxidants produces robust and long-lasting slices, but does not have any apparent effect on the pharmacology of glutamate or GABA transmission.

Nevertheless, in both recording situations slices were allowed to equilibrate in the recording chamber for at least 1 hour prior to recording to allow for washout of these agents. The perfusion and storage aCSF contained (in $\mathrm{mM}$ ): $\mathrm{NaCl}$ (126), $\mathrm{KCl}(3.25)$, $\mathrm{NaH}_{2} \mathrm{PO}_{4}$ (1.4), $\mathrm{NaHCO}_{3}(19), \mathrm{MgSO}_{4}$ (2), $\mathrm{CaCl}_{2}$ (2), and D-glucose (10). For cutting the slices at $3-4^{\circ} \mathrm{C}, \mathrm{NaHCO}_{3}$ was increased to $25 \mathrm{mM}$ to maintain $\mathrm{pH}$ at acceptable levels (7.3).

\subsection{Whole-cell patch clamp recordings.}

Patch pipettes pulled from borosilicate glass were used for recording spontaneous EPSCs (sEPSCs). They were filled with a Cs-gluconate based solution containing (in mM) D-Gluconate (100), HEPES (40), QX-314 (1), EGTA (0.6), $\mathrm{MgCl}_{2}$ (5), TEA-Cl (10), phosphocreatinine (5); ATP-Na (4) and GTP-Na (0.3). To record spontaneous (sIPSCs) or miniature (mIPSCs) inhibitory PSCs, the patch solution contained $\mathrm{CsCl}$ (100), HEPES (40), QX-314 (1), EGTA (0.6), TEA-Cl (10), $\mathrm{MgCl}_{2}$ (5), ATP-Na (4) and GTP-Na (0.3). Solutions were adjusted to $275 \mathrm{mOsmol}$ and $\mathrm{pH} 7.3$ with $\mathrm{CsOH}$. Whole-cell voltage clamp recordings (holding potential $-60 \mathrm{mV}$ ) were made from pyramidal neurones in layer III of the medial division of the EC, using an Axopatch 200B amplifier. Signals were filtered at $2 \mathrm{kHz}$ and digitized at $20 \mathrm{kHz}$. Series resistance compensation was not employed, but access resistance (10-30 M $\Omega$ ) was monitored at regular intervals and cells were discarded if it changed by more than $\pm 10 \%$. Liquid junction potentials (EPSC $+12.0 \mathrm{mV}$; IPSCs $+10.2 \mathrm{mV}$ ) were estimated using pClamp-8 software, and compensated for in the holding potentials. When recording IPSCs, AMPA-receptors and NMDA-receptors were blocked with bath applied NBQX and 2-AP5, respectively.

Data were recorded using Axoscope software and Minianalysis (Synaptosoft, Decatur) was used for analysis of PSCs off-line. Spontaneous events were detected using a threshold-crossing algorithm. Cumulative probability distributions of interevent interval (IEI) of spontaneous currents were compared using the Kolmogorov-Smirnoff test (KS). When data were pooled for this analysis, a minimum of 200 events was sampled during a continuous recording period for each neurone 
under each condition. Mean amplitudes, rise times (10-90\%) and total decay times were compared using a paired t-test. In some cases, to gain an overall picture of the effects of ethosuximide on the level of spontaneous inhibition or excitation, we estimated total charge transfer associated with sIPSCs. This is calculated by measuring the area of SIPSCs or SEPSCs, and is directly proportional to the amplitude multiplied by the decay time (Hollrigel \& Soltesz 1997). We determined charge transfer associated with sPSCs in a set time period of 2 min in control and in the presence of the drug. All error values in the text refer to standard error of the mean.

\subsection{VmD estimations}

Sharp electrodes pulled from borosilicate glass and filled with potassium acetate (3M) were used to make intracellular voltage recordings from pyramidal neurones in layer III of the medial EC using an Axoprobe 1A amplifier (Molecular Devices, Sunnyvale CA, USA) in bridge mode. Once membrane potential was stabilised after impalement, estimates of global background excitation $\left(E_{b g}\right)$ and inhibition $\left(l_{b g}\right)$ were derived from membrane potential fluctuations using the $\mathrm{VmD}$ method at regular intervals throughout the recordings. This approach was derived by Rudolph et al., (2004) and we have adapted it for recording in EC slices (Greenhill and Jones, 2007). Briefly, neurones were depolarised (for 15-20 s) by injection of two levels of known positive current via the recording electrode. The values of the currents differed from neurone to neurone, but were maintained the same throughout any individual experiment. One level was chosen to elicit a depolarization to within 1-2 $\mathrm{mV}$ of action potential threshold, and the second was adjusted to depolarize the neurone to about half way between this and resting membrane potential. Membrane potential fluctuations at these two levels were fitted to Gaussian distributions (using Prism 4 software, GraphPad, San Diego, USA) and the mean and variance of the membrane potential determined. Leak conductance in each neurone was calculated from the ohmic response produced by a small $(0.1 \mathrm{nA} 100 \mathrm{~ms})$ hyperpolarizing current, injected at resting membrane potential. These parameters, together with mean reversal potentials for AMPA-receptors and $\mathrm{GABA}_{\mathrm{A}} \mathrm{r}$ mediated synaptic responses (derived from preliminary experiments Greenhill and Jones, 2007), allowed us to use the $\mathrm{VmD}$ relationship to quantify background inhibitory and 
excitatory conductances resulting from global network input onto individual neurones.

Cellular excitability was determined by injecting depolarizing current pulses at resting potential during intervals between $E_{b g}$ and $I_{b g}$ estimates. Firstly, action potential (AP) thresholds were determined using brief incremental peri-threshold injections of depolarising current $(0.1-1.0 \mathrm{nA}, 50 \mathrm{~ms})$ via the recording electrode, with firing threshold measured with respect to resting membrane potential. Secondly, trains of action potentials were elicited by longer, supra-threshold current pulses (0.2-1.0 nA, $200 \mathrm{~ms}$ ), and the number of spikes per pulse determined. Action potential amplitudes (from rest) and half-widths were also determined. Statistical analysis (paired $t$-tests or one-way ANOVA) was performed with Prism 4 software. All error values in the text refer to standard error of the mean.

\subsection{Materials}

Salts used in preparation of aCSF were purchased from Merck/BDH or Fisher Scientific (UK). All drugs were applied by bath perfusion. The following drugs were used: ethosuximide (3-ethyl-3-methyl-pyrrolidine-2,5-dione, Sigma-Aldrich, UK), TTX (Alamone Labs, Israel), NBQX (6-nitro-7-sulphamoylbenzo[f]quinoxalone-2,3-dione sodium, Tocris, UK), 2-AP5 (2-amino-5-phosphonopentanoic acid, Tocris, UK).

\section{Results}

The experiments described below were obtained from a total of 40 neurones of the medial division of the EC. Cells for intracellular recordings (the $\mathrm{VmD}$ method) were identified as pyramidal based on their firing characteristics, resting membrane potential and response to injected current, using criteria established by a previous study employing intracellular recordings and biocytin fills [36]. Whole-cell recordings were visualised using IR-DIC optics and cells were selected for their clear pyramidal morphology. Ethosuximide was tested at 2 concentrations, 250 and $500 \mu \mathrm{M}$, which represent the lower and mid-range of the therapeutically relevant plasma concentrations respectively (e.g. see Rogawski and Porter, 1990). 


\subsection{Whole-cell Patch Clamp}

Ethosuximide had little detectable effect on sEPSCs. In control recordings $(n=12)$ the mean IEI was $255 \pm 41 \mathrm{~ms}$ and this was unaltered $(286 \pm 31 \mathrm{~ms})$ in the presence of ethosuximide $(250 \mu \mathrm{M})$. Mean amplitude was also unaltered $(14.1 \pm 1.1 \mathrm{nA} v$ $13.6 \pm 0.9 n A)$. In 7 neurones, ethosuximide was added cumulatively to a concentration of $500 \mu \mathrm{M}$. In these neurones, mean IEI and amplitude were $271 \pm 21$ $\mathrm{ms}$ and $12.9 \pm 0.7 \mathrm{pA}$ in the presence of ethosuximide at $250 \mu \mathrm{M}$, and again neither parameter was altered with the higher concentration $(288 \pm 51 \mathrm{~ms}$ and $12.0 \pm 1.1 \mathrm{pA})$.

Fig. 1A illustrates recordings of SEPSCs in one neurone, and the pooled IEI and amplitude data are shown in Fig. 1B.

In contrast to sEPSCs, ethosuximide caused pronounced changes in sIPSCs. In control conditions, sIPSCs $(n=13)$ had a mean IEI of $115 \pm 21$ ms. Addition of ethosuximide at $250 \mu \mathrm{M}$ decreased IEI to $89 \pm 9 \mathrm{~ms}$. KS analysis of cumulative probability distributions of pooled IEI data showed that the change was significant $(P<0.001)$ and it represented an increase in mean frequency from $9.2 \pm 2.1 \mathrm{~Hz}$ to $11.4 \pm 0.9 \mathrm{~Hz}$. In 6 neurones ethosuximide was increased to $500 \mu \mathrm{M}$, and this resulted in a further decrease in IEI from $90 \pm 13 \mathrm{~ms}$ to $62 \pm 9 \mathrm{~ms}(11.3 \pm 1.2 \mathrm{~Hz}$ to $16.2 \pm 1.0 \mathrm{~Hz})$ and again the change was significant (KS; $\mathrm{P}<0.001)$. Respective mean amplitudes

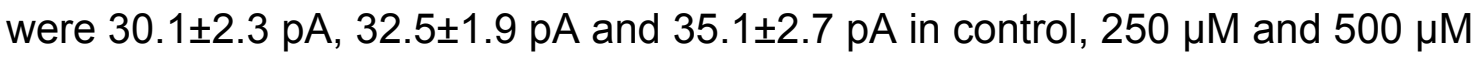
ethosuximide, but the changes were not significant (2-way ANOVA). The drug did not affect rise time of sIPSCs (not shown), and although decay time tended to be slightly longer in the presence of the drug (control 15.3 $\pm 1.6 \mathrm{~ms}$; ethosuximide (250) $16.2 \pm 1.3 \mathrm{~ms}$; ethosuximide (500) $17.4 \pm 1.1 \mathrm{~ms}$ ), the differences were not significant (2-way ANOVA). Recordings in one neurone are illustrated in Fig. 2A. Pooled data for IEI and amplitude are illustrated by the graphs in Fig. $2 \mathrm{~B}$.

To make an overall comparison of the level of spontaneous GABAergic inhibition we estimated the total charge transfer associated with sIPSCs. In control conditions charge transfer in a 2 min recording block was $1620 \pm 281 \mathrm{pC}$, and this increased to $2419 \pm 402 \mathrm{pC}$ and $3420 \pm 495 \mathrm{pC}$ in the presence of ethosuximide at $250 \mu \mathrm{M}$ and 500 $\mu \mathrm{M}$, respectively. The increase at $250 \mu \mathrm{M}$ just failed to reach significance, but that at $500 \mu \mathrm{M}$ was significant $(\mathrm{P}<0.05)$. These data are illustrated in Fig. $2 \mathrm{C}$. 
In 4 neurones we determined the effect of ethosuximide $(500 \mu \mathrm{M})$ on mIPSCs recorded in the presence of TTX $(1 \mu \mathrm{M})$. Mean IEI and amplitude in control recordings was $236 \pm 42 \mathrm{~ms}$, and $22.9 \pm 1.8 \mathrm{pA}$, reflecting a reduced frequency and amplitude of isolated activity-independent release compared to sIPSCs. However, ethosuximide was still effective in elevating GABA release, decreasing mean IEI to $114 \pm 32 \mathrm{~ms}$. Mean amplitude of mIPSCs in the presence of the drug were slightly larger $(25.1 \pm 1.3 \mathrm{pA})$ but, again, the change was not significant.

\section{2. $V m D$ studies}

Thus, ethosuximide increased spontaneous GABA release, but had little effect on spontaneous glutamate release. We next determined whether these changes in spontaneous transmitter release reflected global background inhibition or excitation by using the $\mathrm{VmD}$ approach.

Sharp electrode recordings $(n=6)$ gave an estimated mean global background inhibitory conductance or $\mathrm{I}_{\mathrm{Bg}}$ of $5.8 \pm 0.6 \mathrm{nS}$. In contrast, mean $\mathrm{E}_{\mathrm{Bg}}$, the global background excitation was considerably lower at $1.4 \pm 0.2 \mathrm{nS}$. Thus, the mean I:E ratio was $4.3 \pm 0.3$, which is entirely consistent with previous studies showing a dominance of inhibition over excitation (Greenhill and Jones, 2007; Rudolph et al., $2004 ; 2007)$. In the presence of ethosuximide $(250 \mu \mathrm{M}) \mathrm{I}_{\mathrm{Bg}}$ increased to $9.1 \pm 3.0 \mathrm{nS}$ but this just failed to reach significance. Concurrently $E_{B g}$ was unaltered $(1.4 \pm 0.2 \mathrm{v}$ $1.2 \pm 0.3 \mathrm{nS}$ ). However, despite the lack of significant change in either parameter individually, there was a significant change in the I:E ratio in favour of inhibition from $4.3 \pm 0.3$ to $7.6 \pm 1.3(P<0.05)$. The changes in global conductances and the I:E ratio are summarised in Fig. $3 \mathrm{~A}$ and $\mathrm{C}$.

In 5 further neurones we tested the effect of ethosuximide at $500 \mu \mathrm{M}$ (Fig. 3B-D). In control conditions $\mathrm{I}_{\mathrm{Bg}}$ was $4.5 \pm 0.5 \mathrm{nS}$ and $\mathrm{E}_{\mathrm{Bg}}$ was estimated at $1.3 \pm 0.1 \mathrm{nS}$, giving a mean I:E ratio of $3.7 \pm 0.3$. With the addition of ethosuximide, $E_{B g}$ remained stable at $1.2 \pm 0.2 \mathrm{nS}$, but $\mathrm{I}_{\mathrm{Bg}}$ increased substantially and significantly to $12.1 \pm 2.2 \mathrm{nS}(P<0.05$, paired t-test), resulting in a marked rise in mean $\mathrm{I}: \mathrm{E}$ ratio to $9.8 \pm 1.3$. 
The increase in dominance of global background inhibition over excitation was accompanied by evidence of a reduction in cellular excitability, without any appreciable change in spike generating mechanisms. Control parameters for neurones in studies with either concentration of ethosuximide were similar and so are pooled for simplicity. Mean membrane potential in control conditions was $71.5 \pm 0.7 \mathrm{mV}$. This tended to hyperpolarize with both $250 \mu \mathrm{M}(-73.6 \pm 0.6 \mathrm{mV})$ and 500 $\mu \mathrm{M}$ ethosuximide $(-74.4 \pm 0.8 \mathrm{mV})$, but the changes were not significant. Mean action potential amplitude and half-width were $95.6 \pm 1.2 \mathrm{mV}$ and $0.51 \pm 0.06 \mathrm{~ms}$, respectively. These parameters were also unaltered by ethosuximide at either 250 $\mu \mathrm{M}(97.7 \pm 1.8 \mathrm{mV}, 0.48 \pm 0.06 \mathrm{~ms})$ or $500 \mu \mathrm{M}(96.6 \pm 2.1 \mathrm{mV}, 0.44 \pm 0.05 \mathrm{~ms})$. Concurrently, however, with the lower concentration, spike firing threshold was increased from $21.6 \pm 0.9 \mathrm{mV}$ to $25.0 \pm 1.2 \mathrm{mV}$ positive to rest $(P<0.05)$, and the number of spikes evoked by a $250 \mathrm{~ms}$ depolarising pulse was reduced from $4.8 \pm 0.2$ to $3.0 \pm 0.5(P<0.01)$. The changes were increased further with the higher concentration of ethosuximide, with spike threshold elevated to $27.1 \pm 1.2 \mathrm{mV}$ positive to rest, and the number of spikes evoked by a depolarizing pulse falling to $2.4 \pm 0.6$. The changes in excitability are illustrated for pooled data and in an individual neurone in Fig. 3E and F.

\section{Discussion}

Ethosuximide increased the frequency of sIPSCs in principal neurones in layer III. A small increase in amplitude and decay time of SIPSCs may indicate a weak effect at postsynaptic GABA receptors, but it is likely that the predominant effect of the drug is presynaptically to increase GABA release. The effect was specific to inhibitory synapses as sEPSCs were unaltered by ethosuximide. The increase in GABA release was reflected by an increase in network driven, global background inhibition estimated by the $\mathrm{VmD}$ approach. These studies provide a further validation of $\mathrm{VmD}$ estimations for recording integrated activity in neurones embedded in an active synaptic network (Rudolph et al., 2004; Greenhill and Jones, 2007). The combined observations demonstrate that increased GABA release at the cellular level is reflected by an elevation of global background inhibition, and a shift in balance of network bias to reduce excitability. 
We have previously examined the effects of other anticonvulsants on spontaneous background synaptic activity using the same approaches (Cunningham et al, 2000; 2003; 2004; Cunningham and Jones, 2000; Yang et al, 2007; Greenhill and Jones, 2010) and the profile of effects varied. Phenytoin, lamotrigine and carbamazepine decreased background excitation and concurrently increased inhibition (Cunningham et al., 2000; Cunningham and Jones, 2000; Greenhill and Jones, 2010), all three drugs increasing I:E ratio. In contrast, gabapentin, pregabalin and felbamate decreased excitation but left inhibition intact (Cunningham et al., 2004; Yang et al., 2007; Greenhill and Jones, 2010), again increasing I:E ratio. Valproate decreased the frequency of both sEPSCs and sIPSCs in whole cell patch clamp studies but it prolonged the decay time of sIPSCs so, overall, synaptic inhibition and $\mathrm{I}_{\mathrm{Bg}}$ were effectively increased (Cunningham et al., 2000; Greenhill and Jones, 2010) with a consequent increase I:E ratio. Finally, the GABA uptake blocker, tiagabine decreased SIPSC frequency but increased both amplitude and decay time, again effectively increasing $\mathrm{I}_{\mathrm{Bg}}$. It concurrently increased sEPSC frequency and, thus, weakly increased $E_{\mathrm{Bg}}$ (Greenhill and Jones, 2010), but overall, it also increased I:E ratio. The current observations reveal a new spectrum of effects with ethosuximide, but again, as with the other drugs investigated, I:E ratio was increased.

Thus, despite the varying profile of effects and regardless of the (putative) molecular targets of the different drugs (e.g. see Loscher and Rogawski, 2004; White et al., 2007) they all increased global I:E ratio demonstrating a biasing of global network activity in favour of inhibition. Concurrently, cellular excitability was decreased by all the anticonvulsants (Greenhill and Jones, 2010), although any connection between these effects remains circumstantial at present. However, there is a considerable literature suggesting that background network activity is instrumental in determining neuronal excitability and gain (see Jones and Woodhall, 2005; Greenhill and Jones, 2007; and refs therein). Thus, it is possible that a common end point of therapeutic intervention is to decrease neuronal excitability and synchronisation, irrespective of the primary molecular target. It is interesting that somatosensory cortical neurones in GAERS display a depolarized membrane potential and high firing rate, and ethosuximide, concurrent with abolition of spike-wave discharges, restored membrane potential and firing rate to that seen in normal control animals (Polack and Charpier, 2009). In our experiments, ethosuximide tended to hyperpolarize EC 
neurones (but not significantly) and we did see a clear reduction in excitability. Thus, it is tempting to speculate that the reduced excitability seen with ethosuximide in the GAERS (Polack and Charpier, 2009) may be linked to increased background release of GABA.

Do our observations in concord with previous studies of the effect of ethosuximide on glutamate and GABA systems? Early evidence suggested that the reduction in GABA levels subsequent to GAD inhibition in mouse brain could be reversed beyond control levels by ethosuximide (Löscher and Frey, 1977). Ethosuximide alone increased rat whole brain and frontal cortical GABA-levels (Linn-Michell et al., 1986; Ponnusamy and Pradhan, 2006), which could accord with an increased release of GABA. However, it had no effect on spontaneous or stimulated release of GABA in rat cortical slices (Skeritt and Johnson, 1983; Crowder and Bradford, 1987) and did not alter extracellular GABA concentrations in motor cortex in vivo (Terzioglu et al., 2006). Although these studies conflict with the increase in GABA release that we see, they used large-scale approaches to look at release, and may not detect subtle changes at the cellular level. Some evidence also suggests that high concentrations of ethosuximide may weakly block $\mathrm{GABA}_{\mathrm{A}} \mathrm{r}$ in cortical and thalamic neurones (Barnes and Dichter, 1984; Coulter et al., 1990), which contrasts with the trend towards an increased amplitude of mIPSCs and sIPSCs seen in our studies. Finally, there is evidence that ethosuximide may block GABA uptake (Rainesalo et al., 2004). Such an effect could conceivably be reflected as an increase in GABA release at inhibitory synapses (cf tiagabine; Greenhill and Jones, 2010). As with GABA, glutamate release in cortical slices and in motor cortex in vivo was unaffected by ethosuximide (Skerrit and Johnson, 1983; Crowder and Bradford, 1987; Terzioglu et al., 2006), and chronic administration of the drug had no effect on glutamate levels in frontal cortex or hippocampus (Ponnusamy and Pradhan, 2006). These results agree with ours that suggest that glutamate release is unaffected by ethosuximide (but see discussion concerning Huang et al. (2011) below).

We have not studied the molecular targets of ethosuximide, but some discussion is warranted. It has been suggested that T-type $\mathrm{Ca}^{2+}$ channels in thalamo-cortical neurones are a primary target of ethosuximide (Coulter et al., 1989a,b; Kostyuk et al., 1992; MacDonald and Kelly, 1994; Gomora et al., 2001), blockade of which is 
proposed to reduce oscillatory burst firing and the consequent drive to spike-wave discharges in the cortex. Other evidence suggested that somatic T-type currents are weakly blocked by ethosuximide (Thompson and Wong, 1991; Sayer et al., 1993; Leresche et al., 1998) so such a mechanism of action is equivocal. It is possible that an action at T-type channels could be involved in the effects of ethosuximide on GABA-release. At least one subtype of T-type channel (CaV3.3) is localised to soma and dendrites of calbindin and parvalbumin containing interneurones in mouse somatosensory cortex (Liu et al., 2011). However, if ethosuximide were blocking equivalent channels in the EC we might expect a decreased interneurone excitability and firing, and a consequent decrease in GABA release rather than an increase. Since ethosuximide also increased the activity independent release of GABA, this largely rules out an interaction with somato-dendritic channels. T-type Ca-channels do not appear to be located on presynaptic inhibitory terminals in somatosensory cortex (CaV3.3, Lui et al., 2011) or in layer III of the EC (CaV3.2, Huang et al., 2011) and, in any case, it would be difficult to reconcile a blockade of these channels with the increase in GABA release we observe.

Persistent Na-channels, Ca-activated K-channels and GIRKs (Leresche et al., 1998; Kobayashi et al., 2009) may also be blocked by ethosuximide. Blockade of somadendritic K-channels in interneurones could potentially lead to increased tonic firing and elevated GABA release but the increase in activity independent release seems to rule out this possibility. It is possible that an effect on GIRKs in inhibitory terminals could elicit an increase in GABA release. For example, we have shown that GABA release at inhibitory terminals in the $E C$ can be depressed by $G A B A_{B}$-receptors (GABA ${ }_{B} r$; Bailey et al., 2004), and this could involve activation of GIRKs. GABA ${ }_{B} r$ can be tonically activated by ambient GABA (Bailey et al., 2004), so if ethosuximide was to block the GIRK linked to this receptor, it could result in a reduction in tonic depression of GABA release, manifested as an increase. Such a possibility deserves investigation.

We found no change in glutamate release with ethosuximide, but it should be noted that T-type channels (CaV3.2) have been located at excitatory terminals in layer III of the EC, where they can, under some circumstances, enhance glutamate release (Huang et al., 2011). This study suggests that the channels may be inactivated under 
resting conditions, and may contribute to release only when terminals are hyperpolarized (Huang et al., 2011). Our studies suggest that even if these channels were blocked by ethosuximide this may be of minor consequence under resting conditions, since glutamate release was unaffected. It is possible is that ethosuximide could reduce glutamate release by blocking presynaptic channels when terminals are hyperpolarized, but the circumstances under which this might occur conjectural. Nevertheless, it is interesting that blockade of T-type channels by ethosuximide may be stronger at hyperpolarised potentials (Coulter et al., 1989b).

Our experiments have been conducted in normal tissue so we need to ask is whether the results have relevance to the epileptic situation. Stringer (1996) showed that pentylenetetrazole, which blocks the GABA ${ }_{A}$ (Huang et al., 2001), enhanced the spread of evoked epileptiform activity from the EC to the hippocampus in vivo. The enhancement was attenuated by ethosuximide, and this occurred via an action of the drug on the EC itself (Stringer, 1996). One explanation for this could be that increased GABA release elicited by ethosuximide could overcome the competitive block (Huang et al., 2001) of GABA receptors by pentylenetetrazole. Of profound interest are studies in a mouse model of familial absence epilepsy (Tan et al., 2007). Mice heterozygous for a point mutation in the $\mathrm{GABA}_{A} r \gamma 2$-subunit showed spontaneous cortical spike-wave absence seizures. The seizures were accompanied by a reduction in amplitude of mIPSCs in layer $2 / 3$ of the cortex. Although the study did not investigate the effects of ethosuximide on spontaneous inhibition, the drug ameliorated the spike-wave discharges. It seems possible that ethosuximide could be acting by increasing GABA release in similar way to that we report here, thus reversing the decreased cortical inhibition seen in the mutant mice.

It is worth considering some paradoxical aspects of our findings. Ethosuximide is therapeutically specific for generalized absence seizures, and may precipitate or exacerbate tonic-clonic partial and generalized seizures (Perucca et al., 1998; Chaves and Sander, 2005). Yet, in common with drugs that target the latter, it increased the ratio of background synaptic inhibition to excitation in cortical neurones (Greenhill and Jones, 2010). It seems likely that a generalised increase in cortical background inhibition is important for anticonvulsant actions, but therapeutic 
specificity for particular types of epilepsy is also conferred by concurrent actions at other molecular targets and locations. In the case of ethosuximide, a combination of increased cortical inhibition may require concurrent modulation of thalamo-thalamo, cortico-thalamo and thalamo cortical interactions (Manning et al., 2003) for the drug to exert its overall anti-absence effects. 


\section{Acknowledgements}

The study was funded by a University of Bath Scholarship for Stuart Greenhill. 


\section{References}

Armand. V., Gabriel, S., Hoffmann, P., Heinemann, U., Vergnes, M. 1998.

Epileptiform activity and changes in field potential responses induced by low $\left[\mathrm{Mg}^{2+}\right]_{0}$ in a genetic rat model of absence epilepsy. Brain Res. 803, 19-26.

Armand, V., Hoffmann, P., Vergnes, M., Heinemann, U. 1999. Epileptiform activity induced by 4-aminopyridine in entorhinal cortex hippocampal slices of rats with a genetically determined absence epilepsy (GAERS). Brain Res. 841, 62-69.

Bailey, S.J., Dhillon, A., Woodhall, G.L., Jones, R.S.G., 2004. Lamina-specific differences in GABA(B) autoreceptor-mediated regulation of spontaneous GABA release in rat entorhinal cortex. Neuropharmacology. 46, 31-42.

Barnes, D.M., Dichter, M.A., 1984. Effects of ethosuximide and tetramethylsuccinimide on cultured cortical neurons. Neurology. 34, 620-625.

Caraballo, R.H., Fontana, E., Darra, F., Bongiorni, L., Fiorini, E., Cersosimo, R., Fejerman, N., Bernardinab, B.D. 2008. Childhood absence epilepsy and electroencephalographic focal abnormalities with or without clinical manifestations. Seizure. 17, 617-624.

Carçak, N., Ferrandon, A., Koning, E., Aker, R.G., Ozdemir, O., Onat, F.Y., Nehlig, A. 2009. Effect of stage 2 kindling on local cerebral blood flow rates in rats with genetic absence epilepsy. Epilepsia. 50, 33-43.

Chaves, J., Sander, J.W., 2005; Seizure aggravation in idiopathic generalized epilepsies. Epilepsia. 46 Suppl 9, 133-139.

Coulter, D.A., Huguenard, J.R., Prince, D.A., 1989a. Specific petit mal anticonvulsants reduce calcium currents in thalamic neurones. Neurosci. Lett. 98, 74-78.

Coulter, D.A., Huguenard, J.R., Prince, D.A., 1989b. Characterization of ethosuximide reduction of low-threshold calcium current in thalamic neurones. Ann. Neurol. 25, 582-593.

Coulter, D.A., Huguenard, J.R., Prince, D.A., 1990. Differential effects of petit mal anticonvulsants and convulsants on thalamic neurones: GABA current blockade. $\mathrm{Br}$. J. Pharmacol. 100, 807-813.

Crowder, J.M., Bradford, H.F., 1987. Common anticonvulsants inhibit $\mathrm{Ca}^{2+}$ uptake and amino acid neurotransmitter release in vitro. Epilepsia. 28, 378-382.

Crunelli, V., Leresche, N., 2002a. Childhood absence epilepsy: genes, channels, neurons and networks. Nat. Rev. Neurosci. 3, 371-382

Crunelli, V., Leresche, N., 2002b. Block of thalamic T-Type $\mathrm{Ca}^{2+}$ channels by ethosuximide Is not the whole story. Epilepsy Curr. 2, 53-56.

Cunningham, M.O., Dhillon, A., Wood, S.J., Jones, R.S.G., 2000. Reciprocal modulation of glutamate and GABA release may underlie the anticonvulsant effect of phenytoin. Neuroscience, 95:343-351. 
Cunningham, M.O., Woodhall, G.L., Jones, R.S.G., 2003. Valproate modifies spontaneous excitation and inhibition at cortical synapses in vitro.

Neuropharmacology. 45, 907-917.

Cunningham, M.O., Woodhall, G.L., Thompson, S.E., Dooley, D.J., Jones, R.S.G., 2004. Dual effects of gabapentin and pregabalin on glutamate release at rat entorhinal synapses in vitro. Eur. J. Neurosci. 20,1566-1576.

Cunningham, M.O., Jones, R.S.G., 2000. The anticonvulsant, lamotrigine, decreases spontaneous glutamate release but increases spontaneous GABA release in the rat entorhinal cortex in vitro. Neuropharmacology. 39, 2139-2146.

Dhillon, A., Jones, R.S.G., 2000. Laminar differences in recurrent excitatory transmission in the rat entorhinal cortex in vitro. Neuroscience. 99, 413-422.

Glauser, T.A., Cnaan, A., Shinnar, S., Hirtz, D.G., Dlugos, D., Masur, D., Clark, P.O., Capparelli, E.V., Adamson, P.C., 2010. Childhood Absence Epilepsy Study Group. Ethosuximide, valproic acid and lamotrigine in childhood absence epilepsy. N. Engl. J. Med. 362, 790-799.

Gomora, J.C., Daud, A.N., Weiergraber, M., Perez-Reyes, E., 2001. Block of cloned human T-type calcium channels by succinimide antiepileptic drugs. Mol Pharmacol. $60,1121-1132$

Galanopoulou, A.S., 2010. Mutations affecting GABAergic signaling in seizures and epilepsy. Pflugers Arch. 460, 505-523.

Gülhan Aker, R., Tezcan, K., Carçak, N., Sakalli, E., Akin, D., Onat, F.Y., 2010. Localized cortical injections of ethosuximide suppress spike-and-wave activity and reduce the resistance to kindling in genetic absence epilepsy rats (GAERS). Epilepsy Res. 89,7-16.

Greenhill, S.D., Jones, R.S.G., 2007. Simultaneous estimation of global background synaptic inhibition and excitation from membrane potential fluctuations in layer III neurones of the rat entorhinal cortex in vitro. Neuroscience. 147: 884-892.

Greenhill, S.D., Jones, R.S.G., 2010. Diverse antiepileptic drugs increase the ratio of background synaptic inhibition to excitation and decrease neuronal excitability in neurones of the rat entorhinal cortex in vitro Neuroscience. 167, 456-474.

Holmes, M.D., Brown, M., Tucker, D.M., 2004. Are "generalized" seizures truly generalized? Evidence of localized mesial frontal and frontopolar discharges in absence. Epilepsia. 45, 1568-1579.

Holmes, M.D., Quiring, J., Tucker, D.M. 2010. Evidence that juvenile myoclonic epilepsy is a disorder of frontotemporal corticothalamic networks. Neuroimage. 49, 80-93.

Hollrigel, G.S., Soltesz, I., 1997. Slow kinetics of miniature IPSCs during early postnatal development in granule cells of the dentate gyrus. J. Neurosci. 17, 51195128.

Huang, R.Q., Bell-Horner, C.L., Dibas, M.I., Covey, D.F., Drewe, J.A., Dillon, G.H., 2001 Pentylenetetrazole-induced inhibition of recombinant gamma-aminobutyric acid type $A(G A B A(A))$ receptors: mechanism and site of action. J. Pharmacol. Exp. Ther. 298, 986-995. 
Huang, Z., Lujan, R., Kadurin, I., Uebele, V.N., Renger, J.J., Dolphin, A.C., Shah, M.M., 2011. Presynaptic HCN1 channels regulate Cav3.2 activity and neurotransmission at select cortical synapses. Nat. Neurosci. 14, 478-486.

Huguenard, J.R., 1999. Neuronal circuitry of thalamocortical epilepsy and mechanisms of antiabsence drug action. Adv. Neurol. 79, 991-999.

Jones, R.S.G., Woodhall, G.L., 2005. Background synaptic activity in rat entorhinal cortical neurones: differential control of transmitter release by presynaptic receptors. J. Physiol. 562, 107-120.

Kobayashi, T., Hirai, H., lino, M., Fuse, I., Mitsumura, K., Washiyama, K., Kasai S., Ikeda, K., 2009. Inhibitory effects of the antiepileptic drug ethosuximide on G proteinactivated inwardly rectifying K+ channels. Neuropharmacology. 956, 499-506.

Kostyuk, P.G., Molokanova, E.A., Pronchuk, N.F., Savchenko, A.N., Verkhratsky, A.N., 1992. Different action of ethosuximide on low- and high-threshold calcium currents in rat sensory neurones. Neuroscience. 51, 755-758.

Leresche, N., Parri, H.R., Erdemli, G., Guyon, A., Turner, J.P., Williams, S.R., Asprodini, E., Crunelli, V., 1998. On the action of the anti-absence drug ethosuximide in the rat and cat thalamus. J Neurosci. 18, 4842-4853.

Lin-Michell, E., Chweh, A.Y., Swinyard, E.A., 1986. Effect of ethosuximide alone and in combination with gamma-aminobutyric acid receptor agonists on brain gammaaminobutyric acid concentration, anticonvulsant activity and neurotoxicity in mice. J. Pharmacol. Exp. Ther. 237, 486-489.

Liu, X.B., Murray, K.D., Jones, E.G., 2011. Low-threshold calcium channel subunit $\mathrm{Ca}(\mathrm{v}) 3.3$ is specifically localized in GABAergic neurons of rodent thalamus and cerebral cortex. J. Comp. Neurol. 519, 1181-1195.

Lombroso, C.T. 1997. Consistent EEG focalities detected in subjects with primary generalized epilepsies monitored for two decades. Epilepsia. 38, 797-812.

Löscher, W., Frey, H.H., 1977. Effect of convulsant and anticonvulsant agents on level and metabolism of gamma-aminobutyric acid in mouse brain. Naunyn S. Arch. Pharmacol. 296, 263-269.

Luhmann, H.J., Mittmann, T., van Luijtelaar, G., Heinemann, U. 1995. Impairment of intracortical GABAergic inhibition in a rat model of absence epilepsy. Epilepsy Res. 22, 43-51

MacDonald, R.L., Kelly, K.M., 1994. Mechanisms of action of currently prescribed and newly developed antiepileptic drugs. Epilepsia. 35, S41-50.

Macdonald, R.L., Kang, J.Q., Gallagher, M.J., 2010. Mutations in GABA receptor $^{2}$ subunits associated with genetic epilepsies. J. Physiol. 588,1861-1869.

Manning, J.P., Richards, D.A., Bowery, N.G., 2003. Pharmacology of absence epilepsy. Trends Pharmacol. Sci. 24, 542-549.

Manning, J.P., Richards, D.A., Leresche, N., Crunelli, V., Bowery, N.G., 2004. Cortical-area specific block of genetically determined absence seizures by ethosuximide. Neuroscience. 123, 5-9.

Meeren. H., van Luijtelaar, G., Lopes da Silva, F., Coenen, A., 2005. Evolving concepts on the pathophysiology of absence seizures: the cortical focus theory. Arch. Neurol. 62, 371-376. 
Meeren, H.K., Pijn, J.P., van Luijtelaar, E.L., Coenen, A.M., Lopes da Silva, F.H., 2002. Cortical focus drives widespread corticothalamic networks during spontaneous absence seizures in rats. J. Neurosci. 22, 1480-1495.

Nehlig, A., Vergnes, M., Boyet, S., Marescaux, C. 1998. Local cerebral glucose utilization in adult and immature GAERS. Epilepsy Res. 32, 206-12.

Perucca, E., Gram, L., Avanzini, G., Dulac, O., 1998. Antiepileptic drugs as a cause of worsening seizures. Epilepsia. 39, 5-17.

Polack, P.O., Guillemain, I., Hu, E., Deransart, C., Depaulis, A., Charpier, S., 2007. Deep layer somatosensory cortical neurons initiate spike-and-wave discharges in a genetic model of absence seizures. J. Neurosci. 27, 6590-6599.

Polack, P.O., Charpier, S., 2009. Ethosuximide converts ictogenic neurons initiating absence seizures into normal neurons in a genetic model. Epilepsia. 50, 1816-1820.

Ponnusamy, R., Pradhan, N., 2006. The effects of chronic administration of ethosuximide on learning and memory: a behavioural and biochemical study on nonepileptic rats. Behav. Pharmacol. 17, 573-580.

Posner, E.B., Mohamed, K,. Marson, A.G., 2005. A systematic review of treatment of typical absence seizures in children and adolescents with ethosuximide, sodium valproate or lamotrigine. Seizure. 14,117-122.

Rainesalo, S., Eriksson, K., Saransaari, P., Keränen, T., 2004. Uptake of GABA and activity of GABA transaminase in blood platelets from children with absence epilepsy. Neurochem. Res. 29, 1873-1877.

Richards, D.A., Manning, J.P., Barnes, D., Rombola, L., Bowery, N.G., Caccia, S., Leresche, N., Crunelli, V., 2003. Targeting thalamic nuclei is not sufficient for the full anti-absence action of ethosuximide in a rat model of absence epilepsy. Epilepsy Res. 54, 97-107.

Rogawski, M.A. Porter, R.J., 1990. Antiepileptic drugs: pharmacological mechanisms and clinical efficacy with consideration of promising developmental stage compounds. Pharmacol. Rev. 423, 223-86.

Rogawski, M.A., Löscher, W., 2004. The neurobiology of antiepileptic drugs. Nat. Rev. Neurosci. 5, 553-564.

Rudolph, M., Piwkowska, Z., Badoual, M., Bal, T., Destexhe, A., 2004. A method to estimate synaptic conductances from membrane potential fluctuations. J. Neurophysiol. 91, 2884-2896.

Rudolph, M., Pospischil, M., Timofeev, I., Destexhe, A., 2007. Inhibition determines membrane potential dynamics and controls action potential generation in awake and sleeping cat cortex. J. Neurosci. 27, 5280-5290.

Sadleir, L.G., Farrell, K., Smith, S., Connolly, M.B., Scheffer, I.E., 2006.

Electroclinical features of absence seizures in childhood absence epilepsy. Neurology. 67, 413-418.

Sayer, R.J., Brown, A.M., Schwindt, P.C., Crill, W.E., 1993. Calcium currents in acutely isolated human neocortical neurons. J. Neurophysiol. 69, 1596-1606.

Seidenbecher, T., Staak., R, Pape, H.C., 1998. Relations between cortical and thalamic cellular activities during absence seizures in rats. Eur. J. Neurosci. 10, 1103-1112. 
Stringer, J.L., 1996. Ethosuximide specifically antagonizes the effect of pentylenetetrazol in the rat entorhinal cortex. Epilepsy Res. 25, 69-77.

Skerritt, J.H., Johnston, G.A. 1983. Inhibition of amino acid transmitter release from rat brain slices by phenytoin and related anticonvulsants. Clin. Exp. Pharmacol. Physiol. 10, 527-533.

Tan, H.O., Reid, C.A., Single, F.N., Davies, P.J., Chiu, C., Murphy, S., Clarke, A.L., Dibbens, L., Krestel, H., Mulley, J.C., Jones, M.V., Seeburg, P.H., Sakmann, B., Berkovic, S.F., Sprengel, R., Petrou, S., 2007. Reduced cortical inhibition in a mouse model of familial childhood absence epilepsy. Proc. Natl. Acad. Sci. 104, 1753617541.

Terzioğlu, B., Aypak, C., Onat, F.Y., Küçükibrahimoğlu, E., Ozkaynakçi, A.E., Gören, M.Z., 2006. The effects of ethosuximide on amino acids in genetic absence epilepsy rat model. J. Pharmacol. Sci. 100, 227-233.

Thompson, S.M., Wong, R.K., 1991. Development of calcium current subtypes in isolated rat hippocampal pyramidal cells. J. Physiol. 439, 671-689.

Tucker, D.M., Brown, M., Luu, P., Holmes, M.D. 2007. Discharges in ventromedial frontal cortex during absence spells. Epilepsy Behav. 11, 546-557.

White, H.S., Smith, M.D., Wilcox, K.S., 2007. Mechanisms of action of antiepileptic drugs. Int. Rev. Neurobiol. 81, 85-110.

Yang, J., Wetterstrand, C., Jones, R.S.G., 2007. Felbamate but not phenytoin or gabapentin reduces glutamate release by blocking presynaptic NMDA receptors in the entorhinal cortex. Epilepsy Res. 77, 157-164. 


\section{Figure legends}

Fig. 1: Ethosuximide (ETX) has no effect on spontaneous glutamate release. A. Whole-cell patch-clamp recordings of sEPSCs from a layer III pyramidal cell in the EC. Application of ethosuximide had no effect at either $250 \mu \mathrm{M}$ or $500 \mu \mathrm{M}$. B. Pooled data (at least 200 events in each cell in each recording condition) in 12 neurones in $250 \mu \mathrm{M}$ and 7 neurones with $500 \mu \mathrm{M}$. Control data for the two drug groups have been pooled for simplicity. Cumulative probability for IEI and amplitude distributions show complete overlap, illustrating the lack of effect of the drug.

Fig. 2: Ethosuximide (ETX) increases spontaneous GABA release. A. Whole-cell patch-clamp recordings of sIPSCs from a layer III pyramidal cell in the EC. Ethosuximide caused an increase in frequency of sIPSCs, which was more marked at the higher concentration $(500 \mu \mathrm{M})$. In this neurone there appeared to be an increase in larger amplitude events as well. B. Pooled data (at least 200 events in each cell in each recording condition) reflect these changes, with a concentrationrelated leftward shift in cumulative probability distribution of IEI, reflecting the increased frequency. There was a slight rightward shift in amplitude distribution at $500 \mu \mathrm{M}$, but this did not reach significance. C. The changes in sIPSCs were reflected by a clear increase in the total inhibitory charge transfer, and again this was concentration-related.

Fig 3. Ethosuximide (ETX) increased global background inhibition but not excitation. A. The graphs show mean data for global background synaptic activity. At $250 \mu \mathrm{M}$, ethosuximide non-significantly enhanced background inhibition together with a slight, but again non-significant fall in excitation. B. Ethosuximide at $500 \mu \mathrm{M}$ had similar, but more marked effects, with the increase in $\mathrm{I}_{\mathrm{Bg}}$ reaching significance. $\mathrm{C}$. The combined effect of these changes resulted in a doubling of the I:E ratio in the presence of the drug which was significant at both concentrations. D. The traces are sharp electrode recordings taken at resting potential $\left(E_{m}\right)$ and at two levels of induced depolarization used to determine VmD (see Greenhill and Jones , 2007) E. Concurrent with the 
effects of ethosuximide on global synaptic conductances there was no apparent change in spike generation mechanisms (no effect on amplitude or half width), but spike threshold was significantly increased and the number of spikes elicited by a $200 \mathrm{~ms}$ depolarizing current pulse was reduced. F. Intracellular recordings from one neurone illustrating the excitability changes. On the left, a short depolarizing current pulse was used to elicit a single pulse, and in the presence of ethosuximide this had to be increased to overcome the elevated spike threshold. On the right, equivalent depolarizing pulses elicited a series of 5 spikes in control but only 2 in the presence of the drug. 
Figure 1

A

control
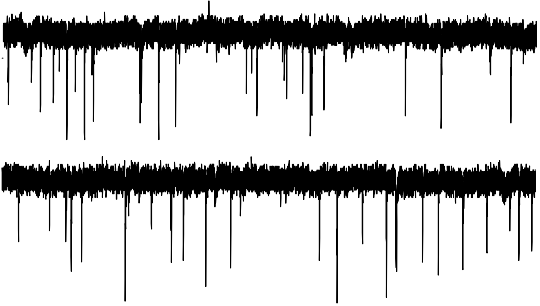

$\operatorname{ETX}(250 \mu \mathrm{M})$
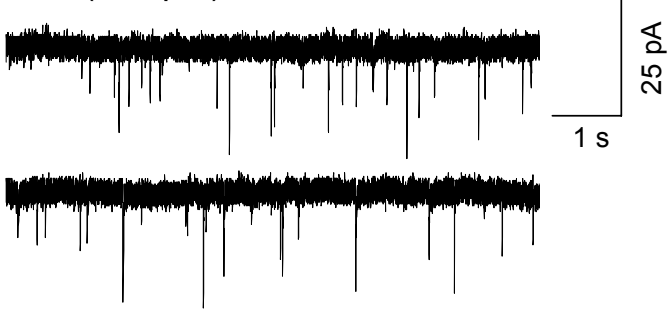

ETX $(500 \mu \mathrm{M})$

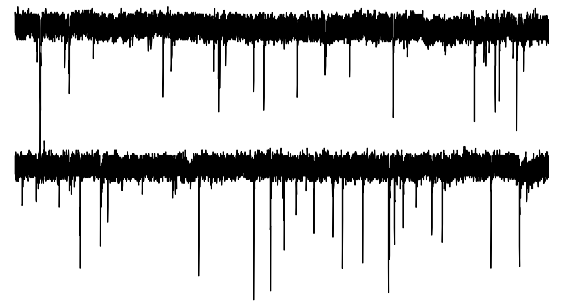

B
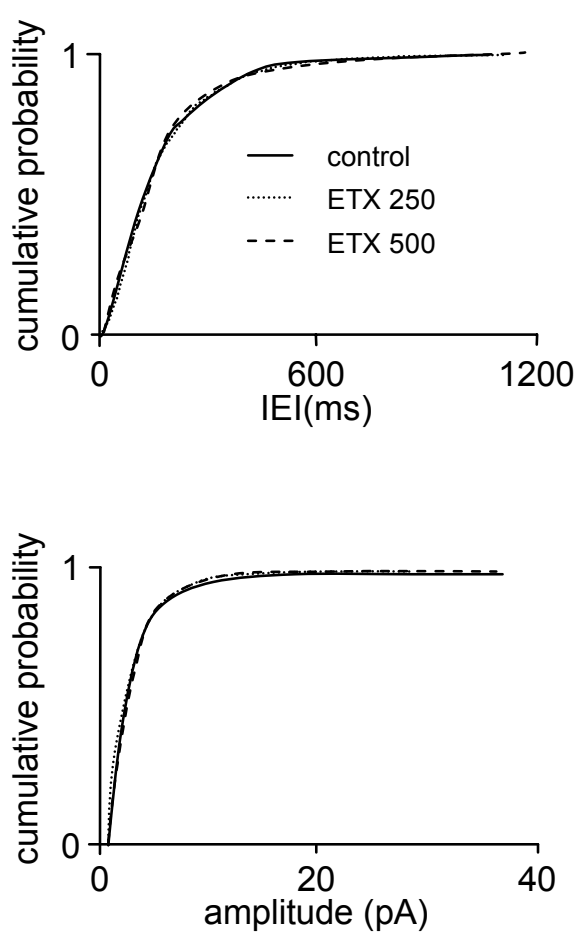
Figure 2

A

control

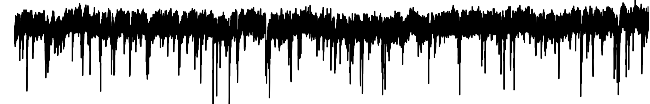

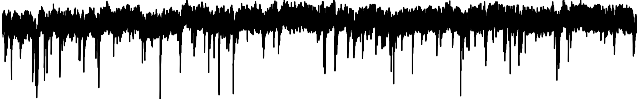

ETX $(250 \mu \mathrm{M})$
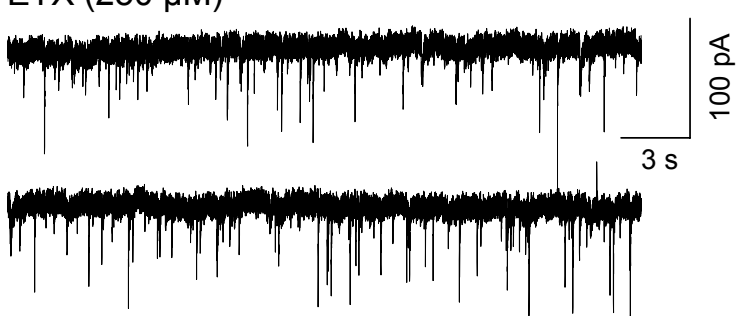

ETX $(500 \mu M)$

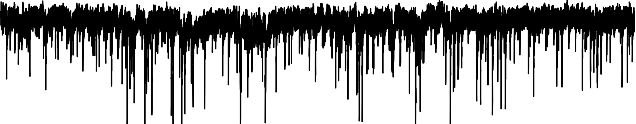

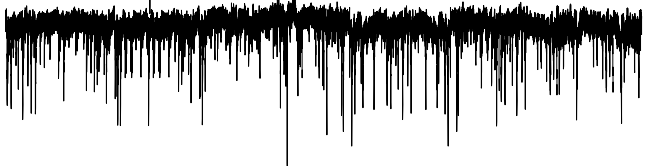

B
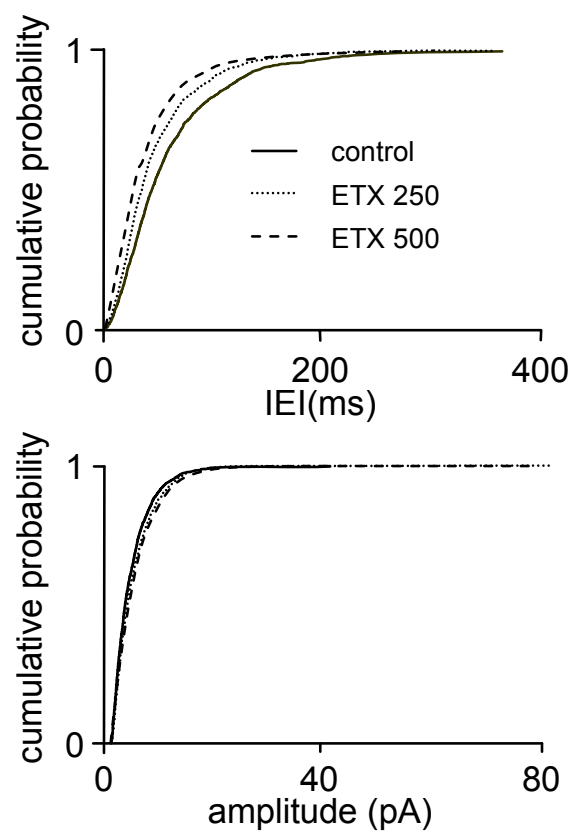

C

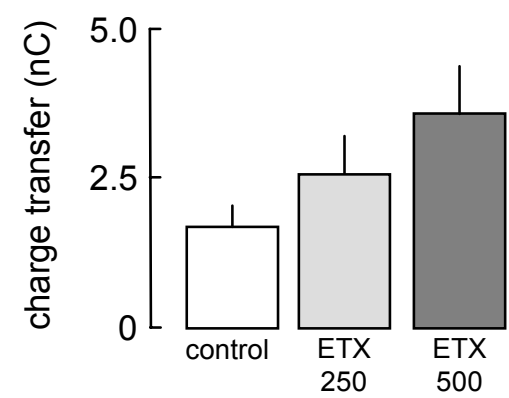


Figure 3

A
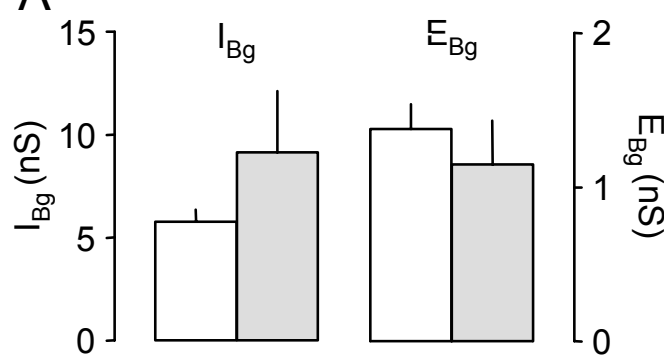

$\square$ control $\square$ ETX $(250 \mu \mathrm{M})$
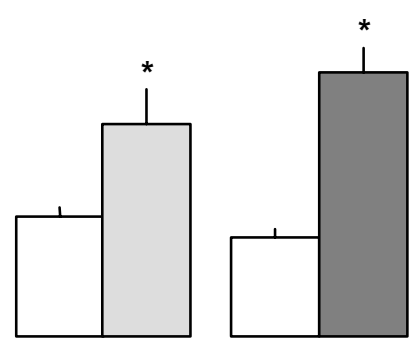

E

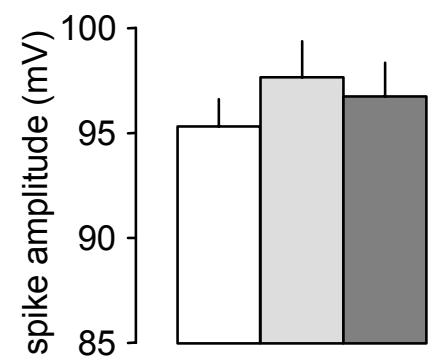

F

control
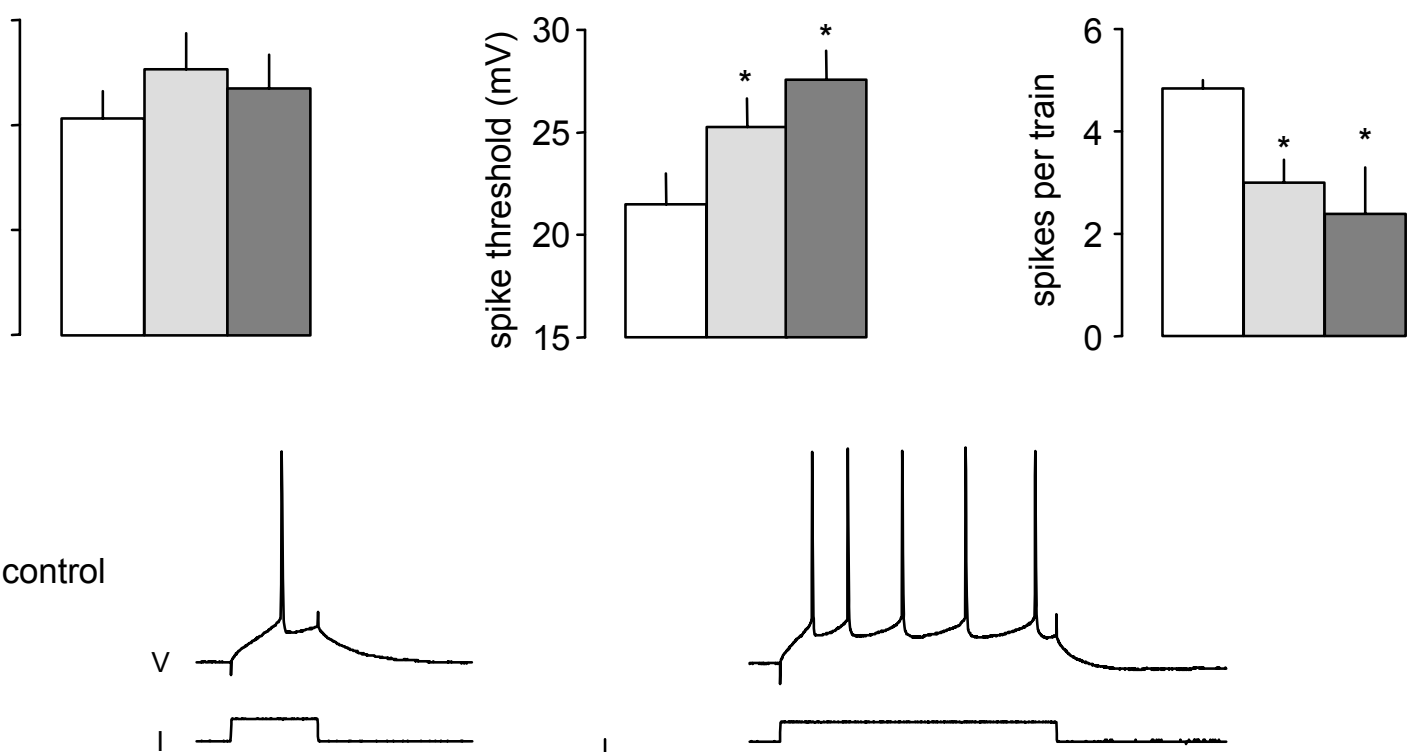

B
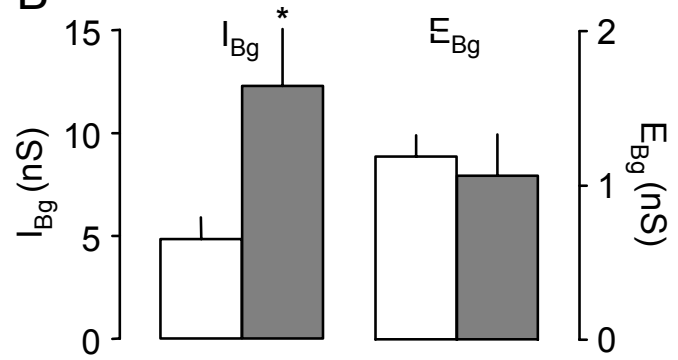

control

ETX $(500 \mu \mathrm{M})$

D

$E_{m}$ $l_{\text {ext1 }}$

$I_{\text {ext2 }}$

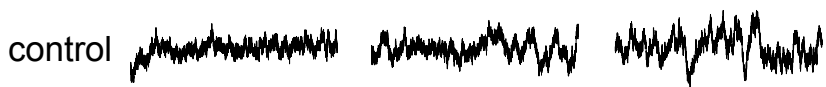

\section{ETX}

$(500 \mu \mathrm{M})$

$\frac{}{2 \mathrm{~s}} 3 \mathrm{mV}$

$F$

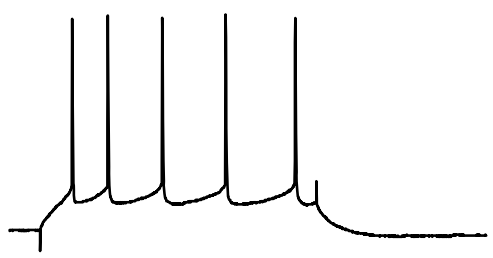

$30 \mathrm{mV}$

$0.5 \mathrm{nA}$

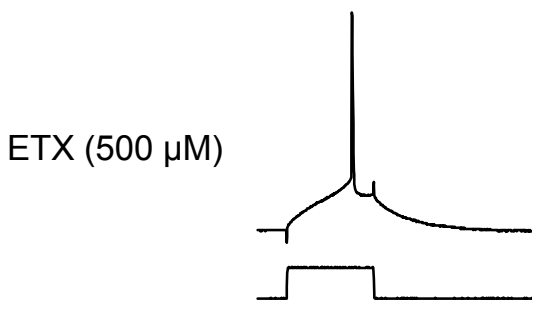

$50 \mathrm{~ms}$

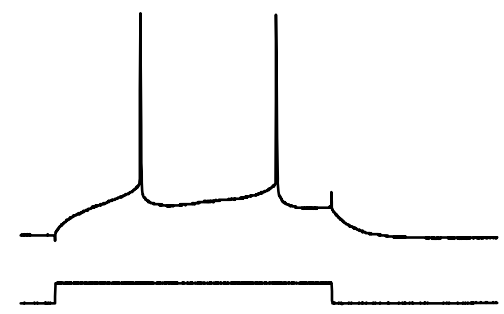

